\title{
An Update on Regional Nodal Irradiation: Indication, Target Volume Delineation, and Radiotherapy Techniques
}

\author{
Marciana Nona Duma \\ Department of Radiotherapy and Radiation Oncology, University Hospital of the Friedrich Schiller University, Jena, \\ Germany
}

\section{Keywords}

Breast cancer · Lymphatics · Radiotherapy · Regional nodal irradiation

\begin{abstract}
Background: Tremendous changes have occurred in the treatment of breast cancer. This paper reviews and unifies the available data on modern axillary management of breast cancer patients with focus on the target volume delineation for regional nodal irradiation according to the most important contouring guidelines, the European Society for Radiotherapy and Oncology (ESTRO) and the Radiation Therapy and Oncology Group (RTOG). Summary: The use of extensive radiotherapy target volumes (level I, II, III, IV) is probably not necessary for all patients to reproduce the clinical benefit shown in the available randomized trials (EORTC, MA.20, AMAROS, Z0011). Nevertheless, given the results in the MA.20 trial, where the patients received more modern systemic therapies and high irradiation doses in the medial paraclavicular region (level IV) and level II, it can be justified to include these regions completely in selected high-risk patients. Key Messages: High-tangent irradiation results in a similar dose distribution in axillary levels I and II compared to the AMAROS treatment field design in some patients. This supports earlier assumptions that irradiation may have accounted for the good results after sentinel lymph node dissection alone in the Z0011 trial. The ESTRO and RTOG clinical target volume (CTV) definitions cover sufficiently the metastatic lymph node hotspots, with a better coverage for the ESTRO CTV. Further, contouring according to the ESTRO would spare a significantly larger part
\end{abstract}

of the healthy lymphatic system, making it our preferred contouring atlas. Modern radiotherapy techniques, such as deep inspiration breath hold, should be cautiously employed in patients treated according to the inclusion criteria of the Z0011 as it will result in a lower dose to the axillary levels.

() 2020 S. Karger AG, Basel

\section{Introduction}

Axillary lymph node dissection (ALND) has a long tradition in breast cancer therapy and was reported as early as 1907 by Halsted [1]. ALND usually implies the removal of $\geq 10$ lymph nodes - located mainly in level I and level II - and has the advantage of reliably identifying nodal metastases. Nonetheless, it is associated with significant morbidity [2]. Further, the impact of ALND on survival has long been disputed. The 25-year findings of the National Surgical Adjuvant Breast and Bowel Project (NSABP) B-04 trial failed to show a significant survival advantage from removing occult positive nodes at the time of initial surgery [3], but meta-analyses demonstrated a benefit [4]. Thus, ALND remained a long-time standard for positive lymph nodes [5]. This changed with the publication of the Z0011 trial, where no differences were found between ALND and no-ALND after positive sentinel nodes. On the other hand, regional node irradiation is a crucial part of the adjuvant therapy in patients with lymph node-positive early breast cancer.

Data from the MA.20 [6] and EORTC 22922-10925 (EORTC) trials [7] have proven that the addition of the 


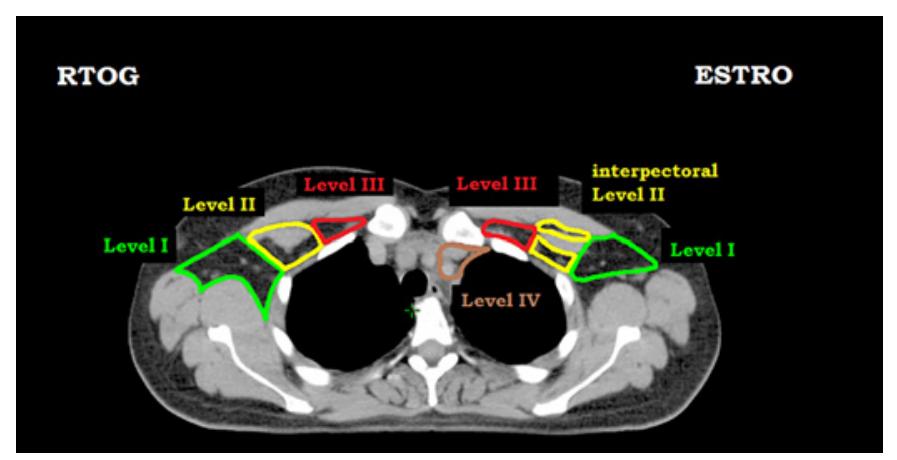

Fig. 1. RTOG and ESTRO contouring recommendations.

supraclavicular and internal mammary lymph node region is effective in improving regional and distant control as well as disease-specific mortality compared to wholebreast or chest-wall irradiation alone.

Furthermore, results from the EORTC 10981-22023 AMAROS trial (AMAROS) revealed that axillary radiotherapy to levels I, II, and III after positive sentinel node biopsy provides excellent axillary control for patients with T1-2 primary breast cancer even when ALND is omitted [8].

In the last decades, tremendous changes occurred in radiation oncology with several new irradiation techniques being employed and routinely used for breast cancer patients. However, with the establishment of computed tomography (CT)-based treatment planning and precise irradiation techniques, definition of the nodal target volume has nowadays become the "weakest part of the quality chain in lymph node radiotherapy" [9]. In order to minimize these uncertainties, contouring atlases were published in 2009 by the Radiation Therapy and Oncology Group (RTOG) (https://www.rtog.org/CoreLab/ ContouringAtlases/BreastCancerAtlas.aspx) and in 2015 by the European Society for Radiotherapy and Oncology (ESTRO). Unfortunately, the atlases differ significantly from each other (Fig. 1).

This paper aims to review the available data on modern axillary management of breast cancer patients with focus on the target volume delineation for regional nodal irradiation according to the most important contouring guidelines, ESTRO and RTOG.

\section{Changes in the Surgical Management of the Axilla}

Sentinel lymph node dissection (SLND) is a minimally invasive procedure for the detection of occult lymph node metastases [10]. The Z0011 trial randomized between SLND and SLND followed by ALND in SLN-positive patients with clinically negative lymph nodes [11]. The study targeted an enrollment of 1,900 women with final analysis after 500 deaths, but the trial closed early because the mortality rate was lower than expected. However, the study changed the treatment approach for SLNpositive patients with clinically negative lymph nodes. Among patients with limited SLN affected, treated with breast conservation and systemic therapy, the use of SLND alone compared with ALND did not result in inferior survival. Thus, patients - if they are within the inclusion criteria of Z0011 - nowadays receive only SLND. Nonetheless, even if the Z0011 protocol required that patients "receive whole breast RT using standard tangential fields without a third field directed to the lymph node areas" a significant proportion of the patients were not irradiated according to the recommendation [12]: half of the analyzed patients had high tangents and approximately $19 \%$ received directed regional nodal irradiation with more than three fields. Most high tangents in the radiotherapy treatment plans covered part of levels I and II of the axilla and thus underlined the importance of radiotherapy to locoregional lymphatics.

\section{Understanding the Radiotherapy Techniques and} the Dosimetry of Randomized Trials on Locoregional Lymphatic Irradiation

All the previously cited radiation oncology studies were launched in the 1990s and early 2000s with very basic radiotherapy techniques (mostly $2 \mathrm{D}$ radiotherapy). The study treatment arms, radiotherapy beam arrangements, and prescribed dose were as follows:

\section{AMAROS}

Patients were randomly assigned to receive either ALND or axillary radiotherapy in the case of a positive sentinel node. ALND and axillary radiotherapy both resulted in excellent and comparable axillary control for patients with T1-2 primary breast cancer and no palpable lymphadenopathy. Axillary radiotherapy resulted in significantly less morbidity.

All three levels of the axilla with the medial part of the supraclavicular fossa were considered as target areas for radiotherapy [8].

The coracoid process was used as a radiological landmark. Treatment of full patient thickness was necessary for levels I and II (lateral to the coracoid process). The target volume of level III could be defined at a depth of 3 $\mathrm{cm}$ from the anterior skin surface. The cranial border was determined by the sternoclavicular joint (which was included with a margin of $3 \mathrm{~cm}$ ), the medial border by the midline of the sternum, and the lateral border by the insertion of the major pectoral muscle at the humerus. The caudal border could be the cranial end of the chest wall or breast fields but had to be at least at the level of the sternal insertion of the second rib (angulus sterni) medially and at the level of the fourth rib laterally. 
An anterior photon field was given with the abovementioned field borders; a smaller posterior-anterior photon field was given with identical field borders except for the medial border, which is situated at the coracoid process. The prescribed dose to the axilla as a whole was $50 \mathrm{~Gy}$ in 25 fractions of $2 \mathrm{~Gy}$.

\section{EORTC}

Women who had a centrally or medially located primary tumor irrespective of axillary involvement and women with an externally located tumor with axillary involvement were included. Patients were randomly assigned to either whole-breast/thoracic-wall radiotherapy and regional nodal irradiation (nodal-irradiation group) or whole-breast/thoracic-wall irradiation alone (control group). Disease-free survival and distant disease-free survival were improved by irradiation of the nodal basin and the breast cancer mortality was reduced.

The target volumes of the group with regional nodal irradiation included the medial supraclavicular and the internal mammary lymphatics [7]. The lateral border was at the middle of the clavicula and the upper border was $3 \mathrm{~cm}$ above the head of the clavicula. The medial border was $1 \mathrm{~cm}$ across the midline but also had to be defined clinically at the supraclavicular region by the patient's radiation oncologist. The lateral field border of the internal mammary part of the field lay $5 \mathrm{~cm}$ lateral from the midline. The lower field border was defined by the lower border of the fourth rib. The lymph node irradiation fields were matched to the tangential fields of the breast.

Anterior-posterior fields were used for the coverage of the lymphatics with a combination of photons and electrons (mixed beam). The prescription point for photons was defined at a depth of $3 \mathrm{~cm}$ on the beam axis. For electrons, the dose was specified at the prescription point, which was defined as the depth of the maximal dose. Doses were applied up to 26 Gy with photons (max. $10 \mathrm{MV}$ ) and up to $24 \mathrm{~Gy}$ with electrons $(12-14 \mathrm{MeV})$ with $2 \mathrm{~Gy}$ per fraction. Thus, the prescribed dose in the target volume was 50 Gy in 2-Gy fractions.

\section{MA.20}

Women with node-positive or high-risk node-negative breast cancer who were treated with breast-conserving surgery and adjuvant systemic therapy were randomized to either whole-breast irradiation plus regional nodal irradiation (including internal mammary, supraclavicular, and axillary lymph nodes) (nodal-irradiation group) or whole-breast irradiation alone (control group). Similar to the EORTC trial, the addition of regional nodal irradiation to whole-breast irradiation did not improve overall survival but reduced the rate of breast cancer recurrence and improved disease-free survival.
The upper internal mammary, supraclavicular, and axillary region (level I-III) nodes were included in the lymphatic treatment field [6]. For this, a 4-field and a modified wide tangent technique ( $50 \mathrm{~Gy} / 2 \mathrm{~Gy}$ ) were used to cover the internal mammary nodes as well as the mammary gland. The breast, internal mammary nodes, and lower axilla were treated with modified wide tangents. The medial border corresponded to a line $3 \mathrm{~cm}$ contralateral to the mid sternum to include the ipsilateral internal mammary nodes as well as the breast. For planning purposes, the lower border of the anterior third intercostal interspace and the lower half of the circumference of the breast was marked with wire. A cardiac and/or lung shield was placed in the inferior, posterior part of the field. Placing of this shield was guided by the outline of the breast and the location of the third intercostal interspace. Below the third interspace the blocking was placed for the medial tangent at the mid sternum. The amount of lung tissue in the upper half of the field had to be $>2 \mathrm{~cm}$ and ideally $<3.5 \mathrm{~cm}$. In the lower half of the field, the amount of lung tissue had to be $<2 \mathrm{~cm}$ to avoid excessive cardiac or lung radiation. The supraclavicular and axillary regions were treated with an anterior field with a prescribed dose at $3 \mathrm{~cm}$ of $50 \mathrm{~Gy}$ (scenario I). An additional posterior field to the axilla was used in all patients with $<10$ nodes removed or $>3$ positive nodes (scenario II). For this scenario the dose was prescribed mid axillary and was $45 \mathrm{~Gy}$ with 1.8 Gy per fraction.

\section{Z0011}

ACOSOG Z0011 randomized women with clinical T1-2 invasive breast cancer, no palpable adenopathy, and 1-2 SLNs containing metastases to undergo ALND after SLND or no further axillary-specific treatment. Among patients with limited SLN metastatic breast cancer treated with breast conservation and systemic therapy, the use of SLND alone compared with ALND did not result in inferior survival.

As previously described, a considerable proportion of the patients, however, received high tangents (defined by cranial tangent border $\leq 2 \mathrm{~cm}$ from the humeral head) [11]. The planning target volume prescribed dose was 50 Gy with 2 Gy per fraction.

In order to treat modern patients, we need to translate all this surgical and radiotherapeutic information into contemporary approaches. Thus, several topics should be revisited and reanalyzed.

\section{Translating Surgical Information into Imaging}

SLNs are mainly located in levels I and II [10]. In patients receiving SLN the drainage of the breast is towards the axilla (levels I and II), the internal mammary chain, interpectoral area, medial and lateral intramammary re- 


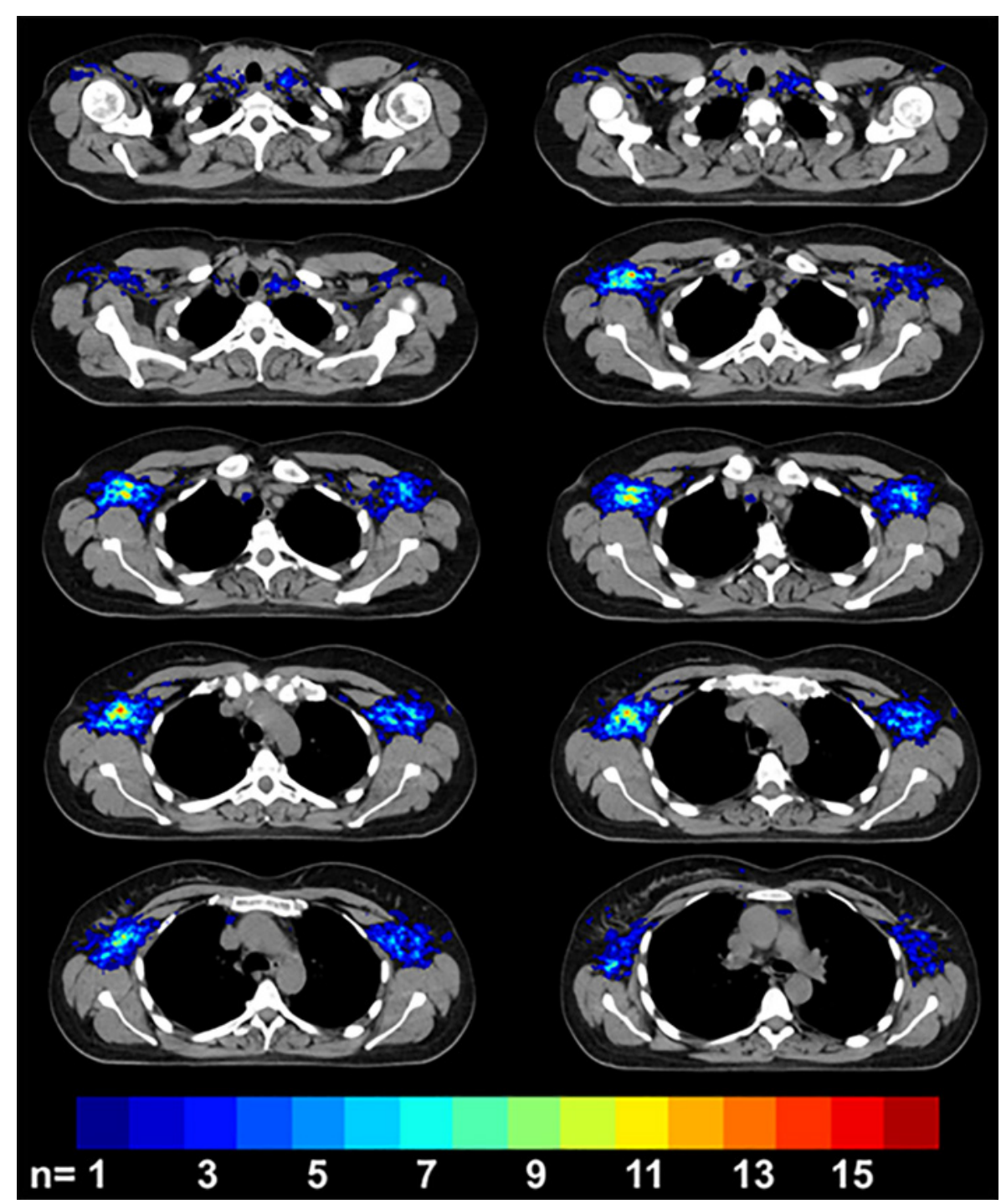

Fig. 2. 3D atlas of location of healthy lymph nodes.

gion, and subclavicular and supraclavicular regions [13]. Nonetheless, in almost $70 \%$ of the cases, SLNs are negative and thus patients would not require an SLND at all [14]. As most patients undergo staging imaging beforehand, reliable information on metastatic lymph nodes would be imperative. However, the surgical/pathological information cannot yet be replaced by imaging information.

We focused on the localization of healthy as well as of pathological lymph nodes in CT and PET-CT imaging. According to our studies, most lymph node metastases occurred in level I dorsolateral to the musculus pectoralis minor and $1-3 \mathrm{~cm}$ below the subclavian vein. Lymph node metastases in levels II, III, and IV accumulated in close proximity to the subclavian vein [15].

"3D lymph node atlases" were created in order to illustrate the physiological and the pathological lymphatic drainage system (Fig. 2, 3) $[15,16]$. Distributions of metastatic and healthy lymph nodes were found to be clearly different within the lymphatic system. Often metastatic lymph nodes occurred outside the regions where healthy lymph nodes were detected by imaging. For example, in levels II and III the overlap between healthy and pathological lymph nodes was outstandingly small [15-17].

\section{Translating Radiotherapy Data of Randomized Trials into 3D Treatment Planning Data}

As previously described, available randomized trials relied mostly on $2 \mathrm{D}$ information. To recreate the $2 \mathrm{D}$ field design on modern CT data sets was imperative [18]. Due to the "simple" dose prescriptions at a specific depth and different techniques used in the randomized studies, significant differences between the studies and between different body shapes (slender patients, obese patients) was found in the coverage of the lymphatics. 

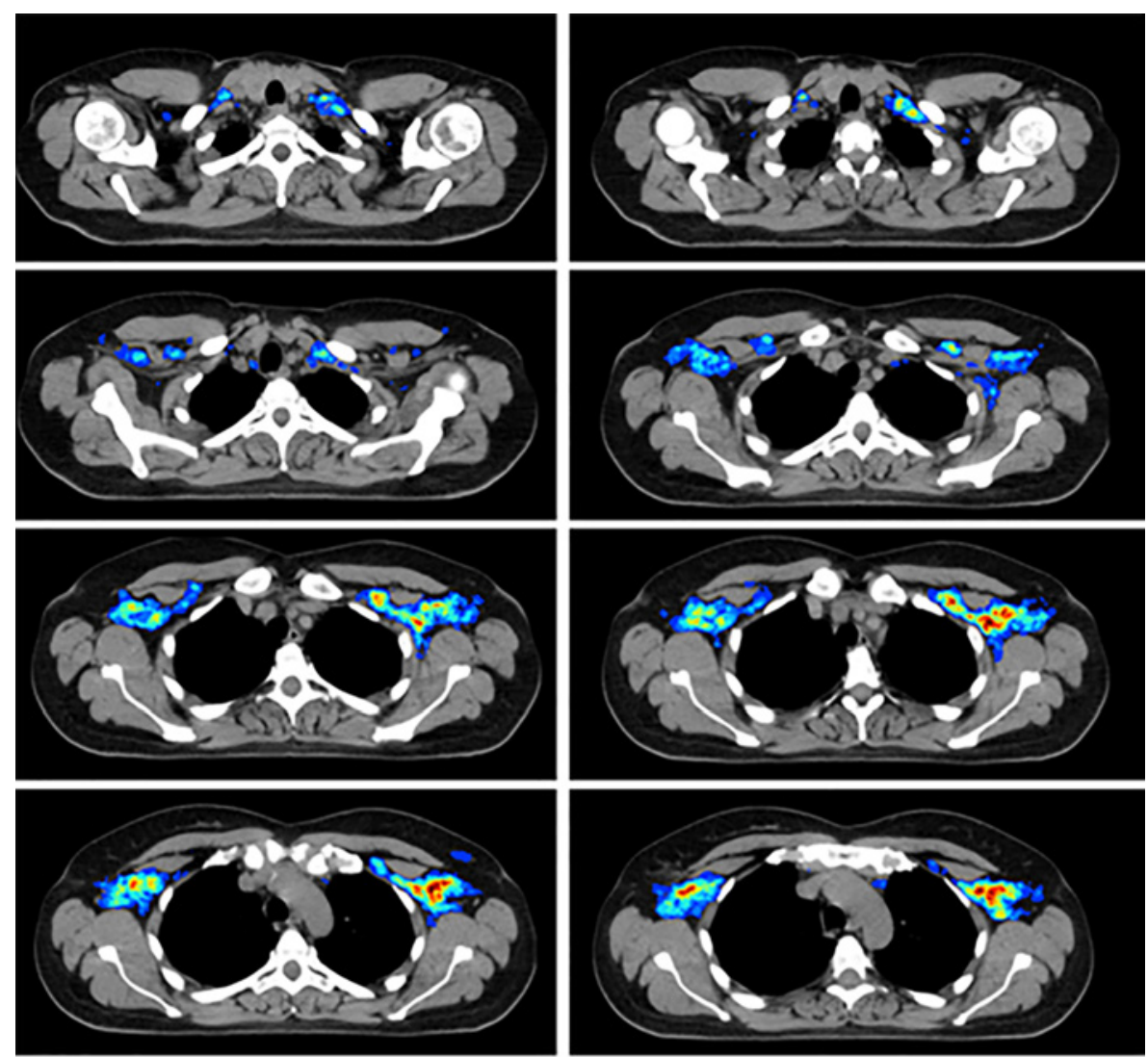

Fig. 3. $3 \mathrm{D}$ atlas of pathological lymph
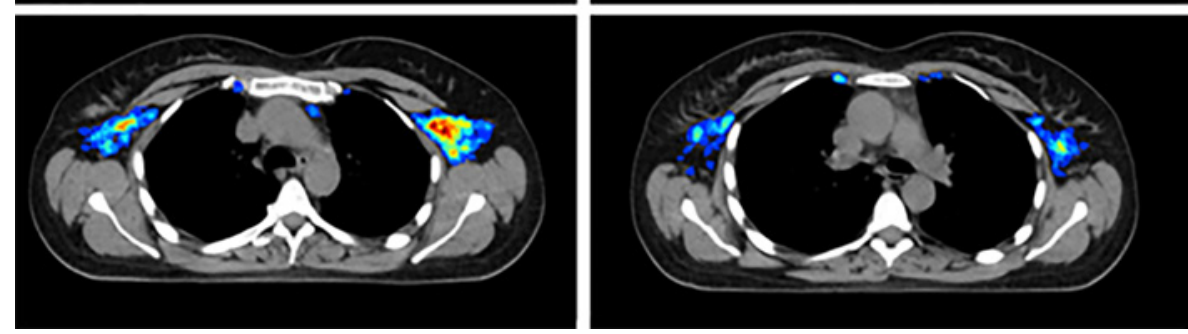
nodes.

Axillary Levels I and II

Treatment of axillary levels I and II was intended in the AMAROS and the MA.20 trial. Most patients in the AMAROS trial would have received the prescribed $50 \mathrm{~Gy}$. As expected, the MA.20 treatment plan without an additional "axilla posterior field" (scenario I) would have led to lower doses in the axillary lymph nodes compared to scenario II (level I: $35.1 \pm 15.8$ Gy vs. $49.9 \pm 4.5$ Gy). However, the doses would also have been dependent on the body shape of the patients. Obese patients would have had a lower dose than the prescribed $45 \mathrm{~Gy}$ in the MA.20 trial (level I: $36.3 \pm 15.2$ Gy). Neither the Z0011 nor the EORTC treatment plans purposely included axillary levels I and II in the target volumes. Correspondingly, the doses for the patients treated in these trials would have been lower compared to the AMAROS and MA.20. Both the Z0011 and the EORTC would - due to the techniques used - have applied an unintended radiotherapy dose to the axilla. The extent of incidental irradiation to the axil- lary nodes would have been highly dependent on the patient's body shape with the lowest values for a slender patient with small breasts [18].

\section{Paraclavicular Lymphatics}

The EORTC, MA.20, and AMAROS trials included the medial supraclavicular fossa in the target volume. However, the dose in the supra-/infraclavicular region would have again varied depending on treatment plan design and patient body shape. We could demonstrate that the best dose coverage would have been for the MA.20 field technique for the slender patient. The EORTC treatment field design led to significantly lower doses compared to the MA.20 field design in the supra-/infraclavicular region. Treatment of the supra-/infraclavicular region was not intended in the Z0011. Correspondingly, dose distribution in the supraclavicular region was negligible in all patients treated similarly to the Z0011 $(<5.0$ Gy). However, relevant incidental irradiation was ob- 
Table 1. Healthy lymph node localization within the ESTRO guideline

\begin{tabular}{|c|c|c|c|c|c|c|}
\hline \multirow[t]{3}{*}{ Lymph node region } & \multicolumn{6}{|c|}{ ESTRO guideline } \\
\hline & \multicolumn{2}{|l|}{ inside } & \multicolumn{2}{|c|}{ partly inside } & \multicolumn{2}{|l|}{ outside } \\
\hline & absolute, $n$ & relative, $\%$ & absolute, $n$ & relative, $\%$ & absolute, $n$ & relative, $\%$ \\
\hline Level I & 521 & 38.8 & 463 & 34.5 & 358 & 26.7 \\
\hline Level II & 115 & 56.4 & 72 & 35.3 & 17 & 8.3 \\
\hline Level III & 8 & 40.0 & 10 & 50.0 & 2 & 10.0 \\
\hline Level IV/supraclavicular & 27 & 9.4 & 50 & 17.4 & 210 & 73.2 \\
\hline Internal mammary & 6 & 11.5 & 8 & 15.4 & 38 & 73.1 \\
\hline Intraglandular & 1 & 2.9 & 0 & 0.0 & 33 & 97.1 \\
\hline Total & 678 & 35.0 & 603 & 31.1 & 658 & 33.9 \\
\hline
\end{tabular}

Table 2. Healthy lymph node localization within the RTOG guideline

\begin{tabular}{|c|c|c|c|c|c|c|}
\hline \multirow[t]{3}{*}{ Lymph node region } & \multicolumn{6}{|c|}{ RTOG guideline } \\
\hline & \multicolumn{2}{|l|}{ inside } & \multicolumn{2}{|c|}{ partly inside } & \multicolumn{2}{|l|}{ outside } \\
\hline & absolute, $n$ & relative, $\%$ & absolute, $n$ & relative, $\%$ & absolute, $n$ & relative, $\%$ \\
\hline Level I & 737 & 54.9 & 383 & 28.5 & 222 & 16.5 \\
\hline Level II & 60 & 29.4 & 71 & 34.8 & 73 & 35.8 \\
\hline Level III & 3 & 15.0 & 8 & 40.0 & 9 & 45.0 \\
\hline Level IV/supraclavicular & 123 & 42.9 & 86 & 30.0 & 78 & 27.2 \\
\hline Internal mammary & 7 & 13.5 & 10 & 19.2 & 35 & 67.3 \\
\hline Intraglandular & 1 & 2.9 & 0 & 0.0 & 33 & 97.1 \\
\hline Total & 931 & 48.0 & 558 & 28.8 & 450 & 23.2 \\
\hline
\end{tabular}

served for the high-tangent treatment plans in the infraclavicular region with doses ranging between $30.0 \pm 19.8$ and $35.3 \pm 19.4 \mathrm{~Gy}[18]$.

\section{Internal Mammary Region}

The MA. 20 and the EORTC treatment arms included irradiation of the internal mammary lymph node region. Accordingly, the lymph nodes were covered by the prescribed doses. A standard tangential field arrangement as performed nowadays in breast cancer would, however, also cover the internal mammary lymph node region with a relevant dose, for example, the MA.20 dose would be $42.5 \pm 14.8 \mathrm{~Gy}$, the EORTC dose would be $39.3 \pm 11.1 \mathrm{~Gy}$, and the 3D-CRT tangential dose would be $30 \pm 17.1 \mathrm{~Gy}$. As before, the anatomy of the patient would also be important: an obese patient would have received a lower dose to the internal mammary compared to a slender patient. The Z0011 and AMAROS treatment plans both led to incidental irradiation of the internal mammary region, with only small differences between standard 3D-CRT tangential and high tangents, but significantly higher doses in the AMAROS treatment plan [18].

\section{Comparison of Available Contouring Guidelines to the Actual Localization of Lymph Nodes}

As previously described, the ESTRO and the RTOG both provided contouring guidelines for breast cancer (Fig. 1). The differences between the two guidelines are mostly related to the cranial, caudal, and dorsal border of the axillary and supraclavicular levels. Further, the ESTRO guideline - as it based its recommendation on conventional simulator-based radiotherapy - resulted in smaller target volumes. The ESTRO committee pointed to the fact that previous guidelines - including the RTOG guidelines - result in larger treated volumes compared to techniques being employed and routinely used by radiation oncologists.

Some studies are available on the appropriateness and pertinence of the two atlases [19-21]. For example, Gentile et al. [19] analyzed 30 breast cancer patients and found approximately $80 \%$ of lymph nodes to be partly outside the cranial and anterior borders of level I as delineated according to the RTOG. Chang at al. [21], on the other hand, used a "representative" CT in which they manually matched lymph nodes as 5-mm spots. Overall, the RTOG CTV failed to include the location of lymph node metastasis in $17.5 \%$ and the ESTRO CTV in 31.5\%. DeSelm et 
al. [20] performed a similar study with manual mapping of lymph nodes as 5-mm circles. All these studies, however, did not comprehensively take the shapes and sizes of the lymph nodes into account.

In the study performed by our group on PET-CT datasets, the occurrence of lymph node metastases inside the RTOG and ESTRO margins in patients was similar to that in the available literature. Further, both atlases performed similarly with regard to the coverage of lymph nodes. The largest geometric deviations between RTOG/ESTRO CTV contours and lymph node occurrence were measured in the supraclavicular and internal mammary regions [15]. Both the RTOG and the ESTRO CTV sufficiently cover the detected "hot spots" within the lymphatic drainage system, with a better coverage of the hotspots for the ESTRO CTV [15]. Nonetheless, regarding the internal mammary region, a larger proportion of the lymph nodes occurred outside the defined internal mammary CTV in our study compared, for example, to that of Chang et al. [21]. This can be explained by the fact that the lymph nodes in our study were represented in their actual size rather than as a $5-\mathrm{mm}$ spot representing the epicenter.

Nevertheless, an incomplete coverage of lymph nodes does not automatically imply the necessity for larger CTVs in the future. An extension of CTVs must be discussed carefully, as minor changes in the target volume may result in drastic changes of the dose in the organs at risk. Such an organ at risk is the healthy lymphatic system in breast cancer radiotherapy.

The ESTRO CTV does not include a large proportion of healthy lymph nodes: many lymph nodes are either partially outside or outside the CTV (approx. 60\%) [16]. Thus, a large part of the physiological lymphatic flow will not be irradiated if lymphatics are targeted by radiotherapy planned according to the ESTRO CTV (Table 1).

The RTOG encompassed a significantly higher proportion of healthy lymph nodes: $48 \%$ of healthy lymph nodes were completely within and $28.8 \%$ were partly within/outside (Table 2) [22].

\section{Radiotherapy Techniques and Their Implication for Breast Cancer}

Due to the deep inspiration anatomical changes that occur (e.g., an anterior/superior movement of the mammary gland) [23], the aim of our study was to evaluate the movements and doses of lymph node levels I, II, and III for irradiation in free breathing and deep inspiration breath hold (DIBH) and thus to understand the differences in the "accidental" regional nodal irradiation. We found a significant dose reduction due to DIBH in level I [24]. Similar results were reported in irradiation of the breast and lymphatics [25].

\section{Conclusion}

In summary, the use of extensive target volumes is probably not necessary for all patients to reproduce the clinical benefit shown in the available randomized trials. Nevertheless, given the high doses in most of the paraclavicular and level II lymph nodes in the MA.20 trial, inclusion of axillary level II in target volumes and extension of the supraclavicular CTV can be justified in selected high-risk patients.

High-tangent irradiation resulted in a similar dose distribution in axillary levels I and II compared to the AMAROS treatment field design in some patients. This supports earlier assumptions that irradiation may have accounted for the good results after SLND alone in the Z0011 trial.

The ESTRO and RTOG CTV definitions sufficiently cover the metastatic lymph node hotspots, with a better coverage for the ESTRO CTV. Further, contouring according to the ESTRO would spare a significant larger part of the healthy lymphatic system, making it our preferred contouring atlas.

Modern radiotherapy techniques, such as DIBH, should be cautiously employed in patients treated according to the inclusion criteria of the Z0011 as it will result in a lower dose to the axillary levels.

\section{Disclosure Statement}

The author has no conflict of interest to declare.

\section{Funding Sources}

The author has no funding sources to disclose.

\section{References}

1 Halsted WS. I. The results of radical operations for the cure of carcinoma of the breast. Ann Surg. 1907 Jul;46(1):1-19.

2 Ivens D, Hoe AL, Podd TJ, Hamilton CR, Taylor I, Royle GT. Assessment of morbidity from complete axillary dissection. Br J Cancer. 1992 Jul;66(1):136-8.

3 Fisher B, Jeong JH, Anderson S, Bryant J, Fisher ER, Wolmark N. Twenty-five-year follow-up of a randomized trial comparing radical mastectomy, total mastectomy, and total mastectomy followed by irradiation. N Engl J Med. 2002 Aug;347(8):567-75.

4 Orr RK. The impact of prophylactic axillary node dissection on breast cancer survival-a Bayesian meta-analysis. Ann Surg Oncol. 1999 Jan-Feb;6(1):109-16.

5 Lyman GH, Giuliano AE, Somerfield MR, Benson AB 3rd, Bodurka DC, Burstein HJ, et al.; American Society of Clinical Oncology. American Society of Clinical Oncology guideline recommendations for sentinel lymph node biopsy in early-stage breast cancer. J Clin Oncol. 2005 Oct;23(30):7703-20. 
6 Whelan TJ, Olivotto IA, Parulekar WR, Ackerman I, Chua BH, Nabid A, et al.; MA.20 Study Investigators. Regional nodal irradiation in early-stage breast cancer. $\mathrm{N}$ Engl J Med. 2015 Jul;373(4):307-16

7 Poortmans PM, Collette S, Kirkove C, Van Limbergen E, Budach V, Struikmans H, et al.; EORTC Radiation Oncology and Breast Cancer Groups. Internal mammary and medial supraclavicular irradiation in breast cancer. $\mathrm{N}$ Engl J Med. 2015 Jul;373(4):317-27.

8 Donker M, van Tienhoven G, Straver ME, Meijnen P, van de Velde CJ, Mansel RE, et al. Radiotherapy or surgery of the axilla after a positive sentinel node in breast cancer (EORTC 10981-22023 AMAROS): a randomised, multicentre, open-label, phase 3 non-inferiority trial. Lancet Oncol. 2014 Nov; 15(12):1303-10.

9 Offersen BV, Boersma LJ, Kirkove C, Hol S, Aznar MC, Biete Sola A, et al. ESTRO consensus guideline on target volume delineation for elective radiation therapy of early stage breast cancer. Radiother Oncol. 2015 Jan;114(1):310.

10 Giuliano AE, Kirgan DM, Guenther JM, Morton DL. Lymphatic mapping and sentinel lymphadenectomy for breast cancer. Ann Surg. 1994 Sep;220(3):391-8; discussion 398401.

11 Giuliano AE, Hunt KK, Ballman KV, Beitsch PD, Whitworth PW, Blumencranz PW, et al. Axillary dissection vs no axillary dissection in women with invasive breast cancer and sentinel node metastasis: a randomized clinical trial. JAMA. 2011 Feb;305(6):569-75.
12 Jagsi R, Chadha M, Moni J, Ballman K, Laurie F, Buchholz TA, et al. Radiation field design in the ACOSOG Z0011 (Alliance) Trial. J Clin Oncol. 2014 Nov;32(32):3600-6.

13 Smith JA 3rd, Gamez-Araujo JJ, Gallager HS, White EC, McBride CM. Carcinoma of the breast: analysis of total lymph node involvement versus level of metastasis. Cancer. 1977 Feb;39(2):527-32.

14 Weaver DL, Krag DN, Ashikaga T, Harlow SP, O'Connell M. Pathologic analysis of sentinel and nonsentinel lymph nodes in breast carcinoma: a multicenter study. Cancer. 2000 Mar;88(5):1099-107.

15 Borm KJ, Voppichler J, Düsberg M, Oechsner M, Vag T, Weber W, et al. FDG/PET-CTbased lymph node atlas in breast cancer patients. Int J Radiat Oncol Biol Phys. 2019 Mar; 103(3):574-82.

16 Ernst L, Borm KJ, Combs SE, Oechsner M, Düsberg M, Duma MN. EP-1299 Lymphatics in breast cancer: healthy nodes versus metastastic nodes. Radiother Oncol. 2019;133:S711.

17 Voppichler J, Borm KJ, Düsberg M, Oechsner M, Combs SE, Duma MN. Lymphknotenmetastasierungsmuster beim primären Mammakarzinom. Strahlenther Onkol. 2018;194(1): 1-222.

18 Borm KJ, Oechsner M, Dusberg M, Buschner G, Weber W, Combs SE, et al. Irradiation of regional lymph node areas in breast cancer dose evaluation according to the Z0011, AMAROS, EORTC 10981-22023 and MA-20 field design. Radiother Oncol. 2020 Jan;142: 195-201.

19 Gentile MS, Usman AA, Neuschler EI, Sathiaseelan V, Hayes JP, Small W Jr. Contouring Guidelines for the Axillary Lymph Nodes for the Delivery of Radiation Therapy in Breast Cancer: Evaluation of the RTOG Breast Cancer Atlas. Int J Radiat Oncol Biol Phys. 2015 Oct;93(2):257-65.
20 DeSelm C, Yang TJ, Cahlon O, Tisnado J, Khan A, Gillespie E, et al. A 3-dimensional mapping analysis of regional nodal recurrences in breast cancer. Int J Radiat Oncol Biol Phys. 2019 Mar;103(3):583-91.

21 Chang JS, Byun HK, Kim JW, Kim KH, Lee J, Cho Y, et al. Three-dimensional analysis of patterns of locoregional recurrence after treatment in breast cancer patients: validation of the ESTRO consensus guideline on target volume. Radiother Oncol. 2017 Jan;122(1): 24-9.

22 Ernst L, Borm KJ, Dusberg M, Oechsner M, Combs SE, Duma MN. Target volume definition of lymphatic pathways of patients with breast cancer - an analysis of physiological lymphatic pathways. Strahlenther Onkol. 2019;195:S42-3.

23 Oechsner M, Duesberg M, Borm K, Wilkens JJ, Combs SE, Duma MN. EP-1306 gated treatment of left-sided breast cancer: evaluation of lung movement, irradiated volume and mass. Radiother Oncol. 2019;133:S715.

24 Borm KJ, Oechsner M, Combs SE, Duma MN Deep-Inspiration Breath-Hold Radiation Therapy in Breast Cancer: A Word of Caution on the Dose to the Axillary Lymph Node Levels. Int J Radiat Oncol Biol Phys. 2018 Jan; 100(1):263-9.

25 Pazos M, Fiorentino A, Gaasch A, Schönecker S, Reitz D, Heinz C, et al. Dose variability in different lymph node levels during locoregional breast cancer irradiation: the impact of deep-inspiration breath hold. Strahlenther Onkol. 2019 Jan;195(1):13-20. 